\title{
Standoff gas detection, identification and quantification with a thermal hyperspectral imager
}

\author{
M. Chamberland ${ }^{1}$, P. Lagueux ${ }^{1}$, P. Tremblay ${ }^{1}$, S. Savary ${ }^{1}$, \\ M.-A. Gagnon ${ }^{1}$, M. Kastek ${ }^{2}$, T. Piątkowski ${ }^{2}$ \& R. Dulski ${ }^{2}$ \\ ${ }^{1}$ Telops Inc., Canada \\ ${ }^{2}$ Institute of Optoelectronics, Military University of Technology, Poland
}

\begin{abstract}
This paper presents detection and identification of gases using an infrared imaging Fourier-Transform Spectrometer (iFTS). The company Telops has developed an iFTS instrument, the Hyper-Cam, which is offered as a medium or long wave infrared sensor. The principle of operation of the spectrometer and the methodology for standoff gas detection and identification is shown in the paper. The algorithm for gas detection and identification is also shown. The gas detection and identification algorithm is generally based on three key factors: the composition of the analysed pixel, the type of model used to estimate the variability of the target and background spaces, and the model used to describe the pure and mixed pixels. The equation modelling the signal reaching the iFTS caused by the presence of a gas is presented and used with the reference signal obtained without the presence of a gas in iFTS' field of view. Some results of the detection and identification of various types of gases are included in the paper along with quantification of detected gases.

Keywords: standoff gas detection, hyperspectral imaging, remote gas quantification, thermal hyperspectral imager.
\end{abstract}

\section{Introduction}

For various reasons, it is of interest to remotely detect the different gases present in the atmosphere. It might be to monitor the pollution, to protect industrial sites, or for situation awareness in a defense context. Infrared spectroscopy has 
been used for many years now as a remote sensing technique. Thermal hyperspectral imagers open the possibility to apply infrared spectroscopy analysis on each pixel and to produce images of gases in the field of view of the imager. This new technique allows one to detect the presence of gases and to produce images of the clouds, to identify the different gases using the absorption spectral signature, and also to quantify the amount of gas in the cloud.

This paper presents the Hyper-Cam, the thermal hyperspectral imager manufactured by Telops in Canada. After, the description of the instrument, we introduce the physical modelling of radiation transfer which is at the base of the algorithms for gas detection, identification and quantification. The last section presents experimental results using the Hyper-Cam.

\section{The Hyper-Cam}

The Hyper-Cam is a lightweight and compact imaging radiometric spectrometer. The spectra measurements are performed using a Fourier-Transform Spectrometer (FTS) or often called "FTIR". It uses a 320x256 LWIR PV-MCT focal plane array detector that can be windowed and formatted to fit the desired size and to increase the measurement rate. Spectral resolution is user selectable and ranges from 0.25 to $150 \mathrm{~cm}^{-1}$. This instrument gives the complete spectrum of each pixel in the image, each pixel having an instantaneous field-of-view of $0.35 \mathrm{mrad}$. An afocal telescope can be installed in the fore optics to increase the pixel iFOV to $1.4 \mathrm{mrad}$. This field-portable sensor is shown in Figure 1.

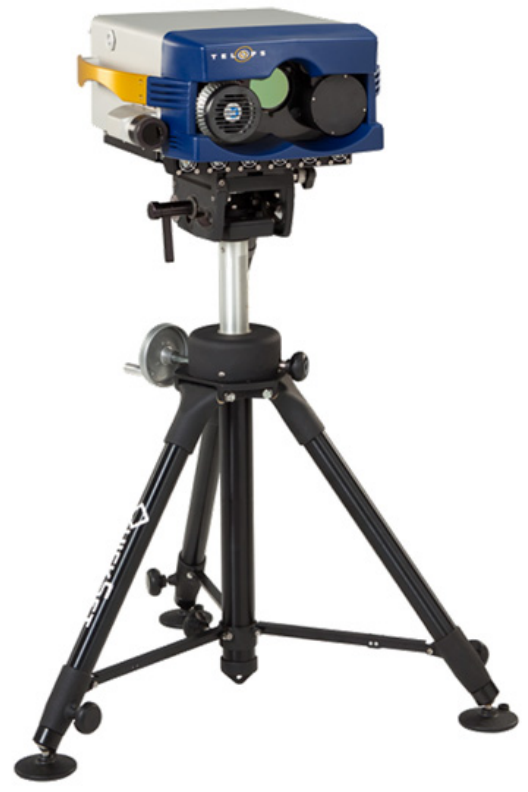

Figure 1: The Hyper-Cam, the Telops' thermal hyperspectral imager. 
The VLWIR version of the Hyper-Cam covers the 8-12 $\mu \mathrm{m}$ infrared band while the MWIR version covers the 3-5 $\mu \mathrm{m}$ band. These 2 bands correspond to the two transparent atmospheric windows, enabling standoff detection of gases. The Hyper-Cam is equipped with two calibration blackbodies which permit to quickly and simply get reference measurements needed to produce calibrated radiance spectra. It is necessary to work with calibrated spectra when performing quantification of the gas clouds.

A personal computer (PC) is connected to the optical head to control the sensor and to get the hyperspectral data. The newest software from Telops to control the Hyper-Cam is "Reveal D\&I". Reveal D\&I is designed specifically for the detection and identification of gas emissions. This powerful software allows real-time detection of a large portfolio of gases simultaneously. A screenshot of Reveal D\&I is presented in Figure 2.

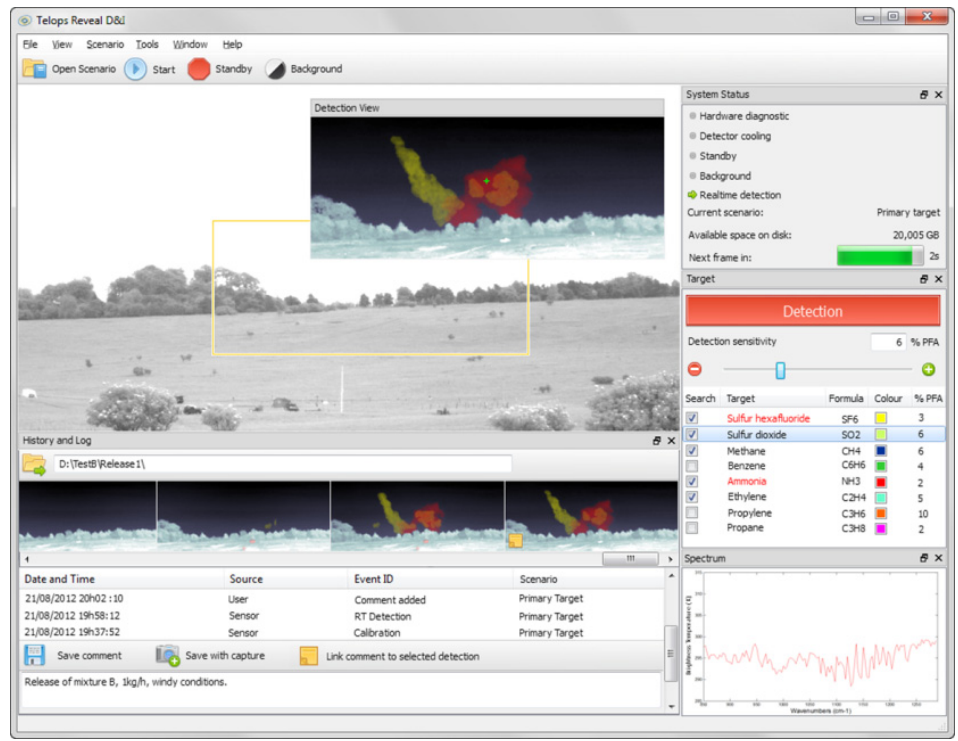

Figure 2: Reveal D\&I software processes in real-time the hyperspectral measurements, displays gas clouds and the gas type.

Hyper-Cams have been used many times for the standoff detection, identification, imaging and quantification of gas clouds [1]. Before presenting some experimental results, we explain the theory of standoff gas detection with this type of sensor.

\section{Standoff detection of gas}

The Hyper-Cam is a passive sensor. It relies on the radiance emitted by the background of the scene and modified by the gas molecules present in the fieldof-view. Figure 3 depicts the physical layout of the measurement topology. For 
each iFOV, the infrared scene may thus be decomposed into 3 distinct contributions [2]. Starting from the instrument, these are a first atmospheric layer, the gas cloud, and the background. The first contribution is made of the atmospheric mass between the plume and the hyperspectral imager. This air mass is here assumed uniform. Next, the gas cloud is including everything getting out of the stack that would otherwise not be present in the atmosphere. Finally, the background represents what is behind the plume, referred at the plume level. The background is here fully characterized by the spectral radiance it shines on the plume in the direction of the hyperspectral imager, since the present model does not include any scattering process.

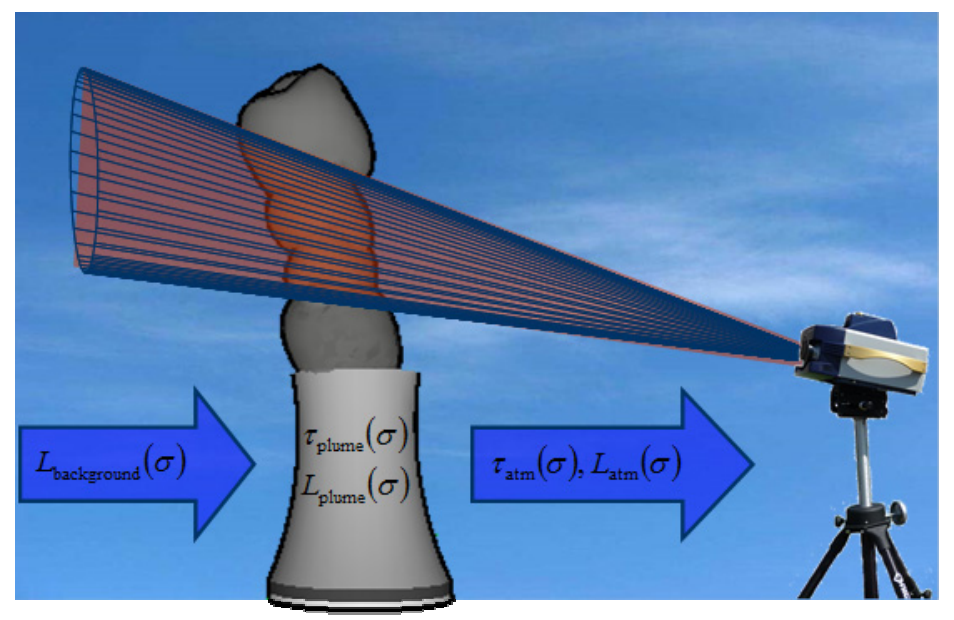

Figure 3: Measurement topology: the hyperspectral imager is looking at the smokestack end. It receives a global spectral radiance whose contributors are the background, the smoke and the atmospheric layer between the stack and the instrument.

The scene is therefore globally represented as a 2-layer model. Starting with a reference surface representing the background radiance, 2 semi-transparent layers are added to model the plume and the atmosphere between the plume and the instrument. When required, the model of the plume itself could be made of multiple layers to better represent the plume temperature non-uniformity across its section. The radiance $L_{\sigma \text {,instr }}(\sigma)$ as measured by the hyperspectral imager can thus be expressed as:

$$
\begin{aligned}
L_{\sigma, \text { instr }}(\sigma)= & L_{\sigma, \text { atm }}(\sigma) \\
& +T_{\text {atm }}(\sigma) \times L_{\sigma, \text { plume }}(\sigma) \\
& +T_{\text {atm }}(\sigma) \times T_{\text {plume }}(\sigma) \times L_{\sigma, \text { background }}(\sigma),
\end{aligned}
$$


where $T_{\text {atm }}(\sigma)$ is the global transmittance of the atmospheric layer between the instrument and the plume and $T_{\text {plume }}(\sigma)$ is the global transmittance of the plume itself (gas cloud). The goal of a quantification algorithm is to estimate the gas species, along with their concentrations, resulting in the latter transmittance. $L_{\sigma \text {,atm }}(\sigma)$ is the total spectral radiance emitted by the atmospheric layer between the instrument and the plume. $L_{\sigma \text {,plume }}(\sigma)$ is the total spectral radiance emitted by the plume itself. $L_{\sigma, \text { background }}(\sigma)$ is the total spectral radiance emitted by the background.

Equation (1) provides the framework to calculate the spectral radiance entering the instrument. It is mainly the first part of the modelling, where the contributions of the atmospheric and plume species are taken into account. The 3 contributions explicitly shown in Equation (1) are illustrated in Figure 4 for a typical scene in the long wave infrared band (LWIR). As expected, the total spectral radiance entering the hyperspectral imager strongly varies across the spectral range. It is interesting to notice that, depending on the wave number, the relative contributions of the 3 sources described in the preceding paragraph, also strongly vary. This shows that none of these 3 contributions may be neglected when one wants to predict the measured radiance across the full spectral range, thus benefiting from every observed piece of information.

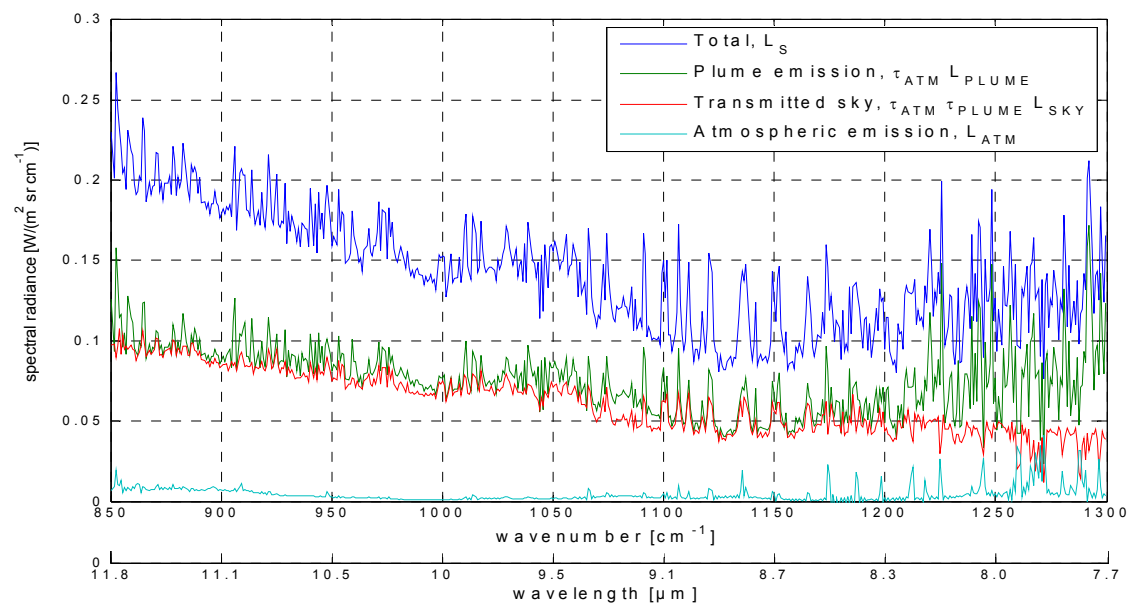

Figure 4: Typical levels of the contributors to the total spectral radiance entering the imaging FTS: transmitted background (sky in this example), transmitted plume emission and atmospheric emission.

The imaging nature of the instrument provides different regions allowing the determination of each contributor. When available, a-priori measurements might also be used to better estimate the background radiance. A library of spectral signatures for the gases of interest is used for the detection and identification algorithm. The spectral signatures consist of absorption spectra for these gases. 
These spectra have been measured by different groups like PNNL [3]. Normalized absorption spectra for 4 typical gases in the VLWIR are presented in Figure 5.

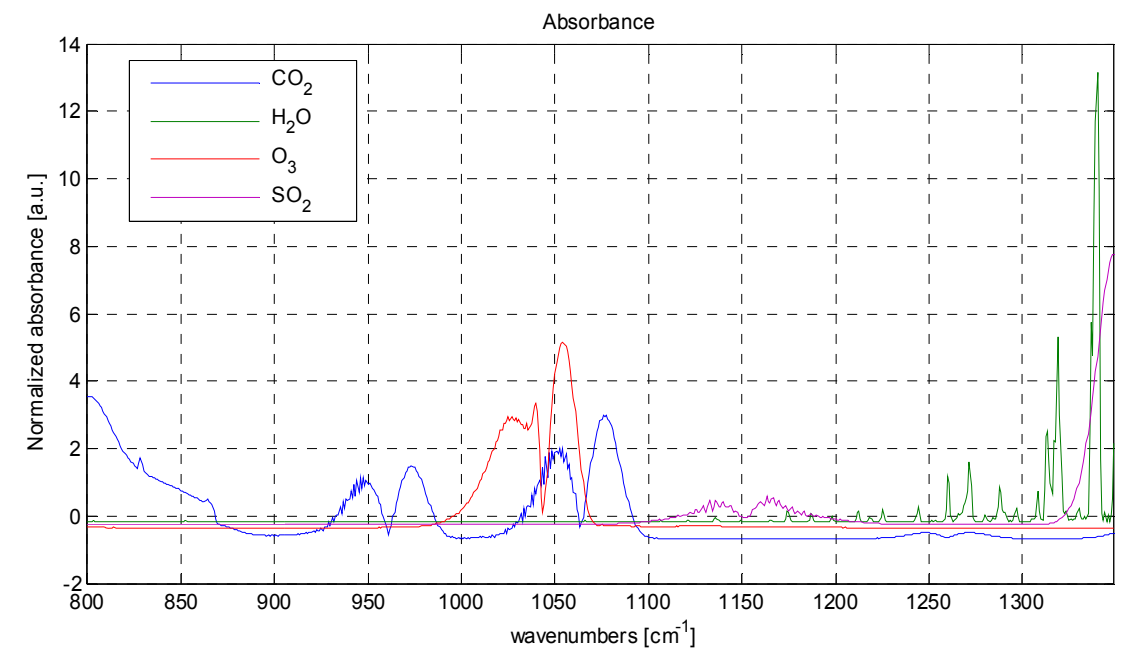

Figure 5: VLWIR absorption spectra (normalized) for $\mathrm{CO}_{2}, \mathrm{H}_{2} \mathrm{O}, \mathrm{O}_{3}$ and $\mathrm{SO}_{2}$. These spectra are presented with a spectral resolution of $1 \mathrm{~cm}^{-1}$.

Each gas shows a distinct spectral signature, often called the "fingerprint". A correlation calculation is made to compare the measured gas cloud spectral radiance with the library of absorption spectra. When correlation gives a good score for a given gas, one gets the detection and identification of that gas in the image. The Telops' Reveal D\&I software displays the gas clouds using a different color for each gas identified (see Figure 2). For the pixels where positive detection and identification occur, it is possible to further analyze the data to determine the quantity of gas in the cloud.

\section{Remote quantification}

In addition to determining the spatial distribution of specific chemicals, it is possible to retrieve a column density value, at each pixel, for each identified species. Absolute quantification, i.e. parts per million ( $\mathrm{ppm})$, at each point in space is not a straightforward procedure from a 2-dimensions image because the infrared signal amplitude is proportional to path length, i.e. the depth (in meters, $\mathrm{m})$ in a scene. Therefore, quantifications are typically expressed in units of $\mathrm{ppm} \times \mathrm{m}$ which represent a column density.

To solve the equation (1), pixels associated to 3 distinct categories have to be identified. First, plume-free pixels have to be selected (on the center of the chimney surface for example) in order to estimate atmospheric parameters (see 
section 4.1 for details). Next, plume-free pixels have to be selected in clear sky areas to enable the calculation of estimates of the background spectral radiance (see section 4.2 for details). Finally, the plume itself has to be localized in the image (see section 4.3 for details). This whole process is depicted in Figure 6.

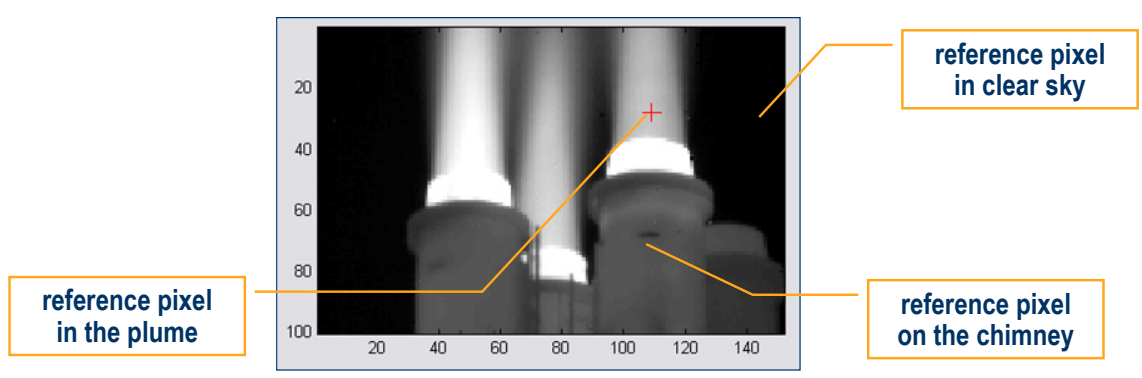

Figure 6: Typical smokestack scene showing distinct areas identified to provide necessary information to achieve plume gas identification and quantification.

\subsection{Atmospheric parameters}

In order to solve Equation (1) to get the plume transmittance, it is first required to determine the transmittance of the atmospheric layer present between the instrument and the smokestack. In this example, this is done by looking at pixels corresponding to the center of the chimney surface (Figure 6).

Retrieval of the atmospheric parameters (temperature and contributing gas species, along their concentrations) is achieved through the use of a set of simplifying hypotheses. First the atmospheric layer is assumed to be uniform, both in temperature and in gas concentrations. To ease solving the problem of retrieving, it is also assumed that the surface of the chimney behaves as a grey body (over the spectral range of the instrument), being fully characterized by its temperature $T_{\text {target }}$ and emissivity $\varepsilon_{\text {target }}$. The measured radiance in these conditions should thus be represented by:

$$
L_{\sigma, \text { instr }}(\sigma)=L_{\sigma, \text { atm }}(\sigma)+T_{\text {atm }}(\sigma) \varepsilon_{\text {target }} L_{\sigma, \mathrm{bb}}\left(\sigma ; T_{\text {target }}\right)
$$

A surface with non-unitary emissivity necessarily reflects part of the radiation it receives. Dealing with hot stacks enables to neglect the reflected radiance.

The core of the retrieval technique is to compare measured spectral radiance with predicted one based on estimated parameters, which are air temperature, concentrations of the selected molecules, temperature of the surface and its emissivity. Selection of given molecules is performed by thresholding estimated concentrations. The Levenberg-Marquardt method is used to find the set of parameters optimizing the least-squares fit. The estimated air temperature and specific molecular concentrations are next used to predict the atmospheric transmittance between the instrument and the plume. 


\subsection{Background radiation}

The next step that must be fulfilled in order to be able to quantify the plume content is to estimate what is the radiance of the plume background. Knowledge of the latter quantity is fundamental since the plume is not opaque over the spectral range, as already shown by Equation (1).

To estimate the background spectral radiance, we select pixels on the same row as the plume pixels for which the quantification has to be done. It is obviously essential to ensure that no plume gas is corrupting the flux received from these directions.

The estimated atmospheric radiance obtained in the preceding step is used to estimate the spectral radiance of the background, reported at the stack level. Here is the relation enabling its calculation:

$$
L_{\sigma, \text { instr }}(\sigma)=L_{\sigma, \text { atm }}(\sigma)+T_{\text {atm }}(\sigma) L_{\sigma, \text { background }}(\sigma)
$$

The method we use to estimate the background spectral radiance implicitly estimates it at the spectral resolution of the hyperspectral imager. No attempt has been made to improve this knowledge.

\subsection{Plume parameters}

From the 2 previous steps, the atmospheric transmittance (and the spectral radiance accounting for the atmospheric emission) and the spectral radiance of the background are known. The only unknown quantities that remain in Equation (1) are the transmittance of the plume and its emitted spectral radiance. In order to proceed further into the quantification, it is here required to determine a plume model. A simple plume model (e.g. a single layer) minimizes the amount of unknown information, thus easing the convergence of the fitting routines, while it may result in inaccuracies due to the use of an inadequate model.

For a single-layer plume model, the spectral emission of the plume is directly obtained from its transmittance. It means that the only unknown quantity that effectively remains in Equation (1) is the transmittance of the plume. The latter depends upon the plume temperature and the concentrations of the selected molecules.

As for the atmospheric transmittance, the core of the retrieval technique is to compare the measured spectral radiance with the predicted one based on the estimated parameters: the atmospheric transmittance, the background spectral radiance, the plume temperature and the concentrations of the selected molecules. The Levenberg-Marquardt method enables to determine the set of parameters optimizing the least-squares fit.

Figure 7 illustrates the estimation of the plume quantities for a single pixel, for a typical scene in the LWIR.

Once the process of getting plume temperature and molecular concentrations (as column densities) is established for a single pixel, it is repeated for neighbouring pixels on the same row of the image in order to obtain plume profiles $[2,4]$. 


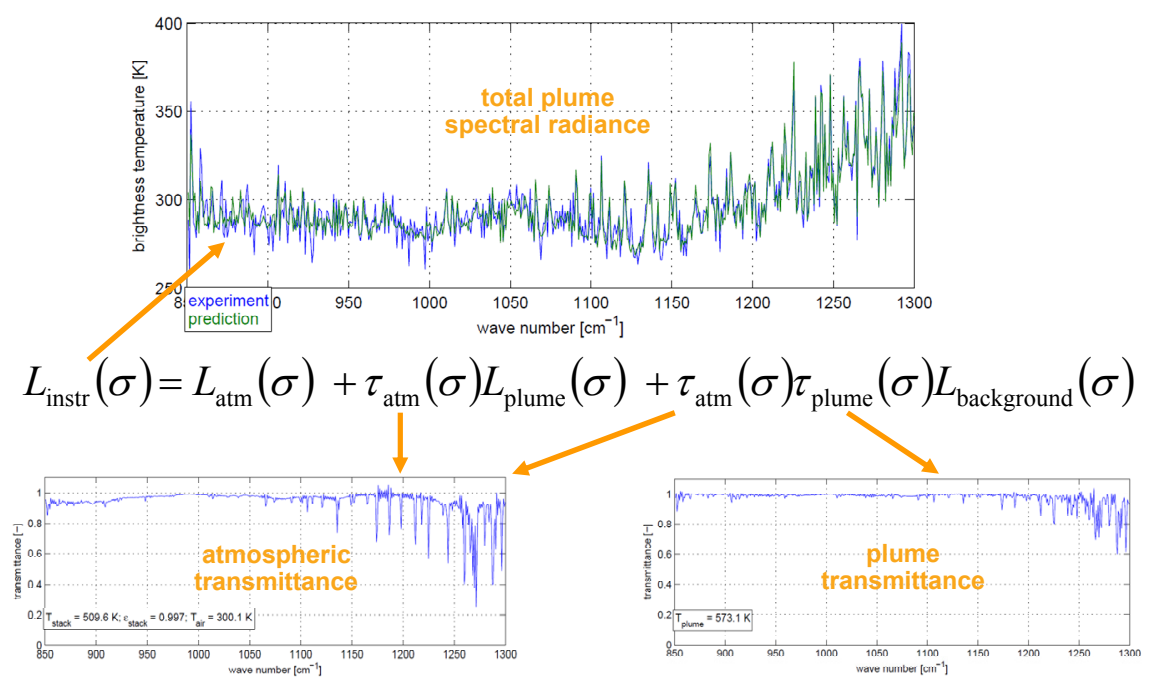

Figure 7: Typical fit of the measured brightness temperature showing the estimated atmosphere and plume transmittances. Fit residuals of the order of $1 \mathrm{~K}$ are routinely achieved.

\section{Results}

We took measurements of the emission of a process vent with a clear sky background. The hyperspectral imager was located at $110 \mathrm{~m}$ from the vent. This experiment is characterized by a relatively small temperature contrast. The performances achieved by the least-squares fit routine are excellent. They are shown in Figure 8 for a typical pixel showing high gas concentrations. The rms residuals are of the order of $0.2 \mathrm{~K}$.

Applying the same process to each pixel in the vicinity of the top of the chimney enables to plot spatial maps of column densities for each of

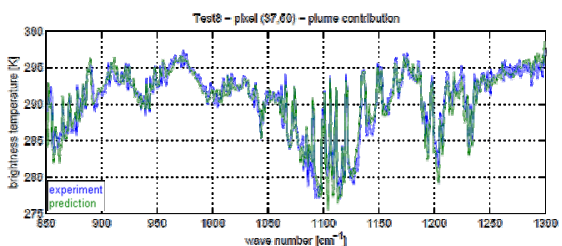

(a)

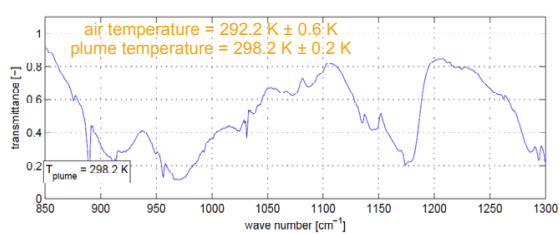

(b)

Figure 8: Estimation of plume transmittance released by a process vent in presence of a low temperature contrast. (a) Agreement of the fit of the estimated spectral radiance when compared to the measured quantity; (b) Estimated infrared transmittance of the semitransparent plume, showing bands where the plume is almost opaque, as well as bands where it is close to transparency. 
the quantified gas. They are presented in Figure 9. Each square pixel shown in the images corresponds to a given pixel of the measured data cube, each one being processed independently of the others.

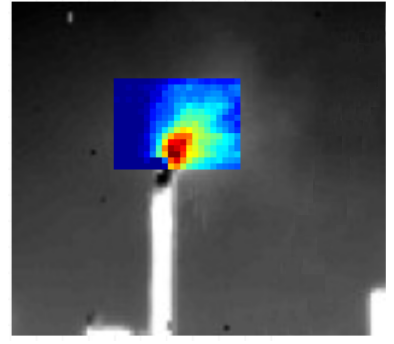

(a)

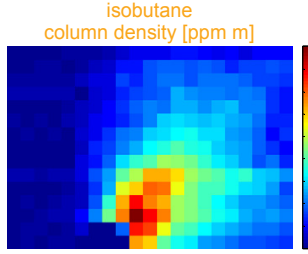

butane

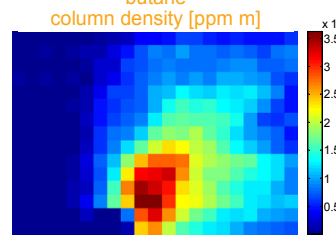

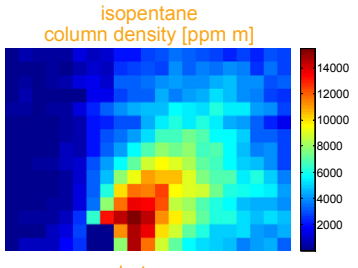

butene

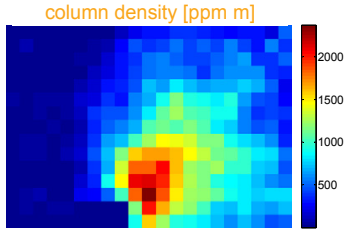

(b)

Figure 9: Gases released by a process vent. (a) Images of brightness temperatures at a selected spectral band; (b) Maps of estimated column densities of some of the identified molecules (isobutene, isopentane, butane and butane).

\section{Conclusion}

Standoff thermal hyperspectral imaging is a powerful approach for characterization of hard-to-reach structures and hazardous environments. Chemical imaging of operating smokestacks and flares shows how information can be obtained easily, rapidly, and without the need of complex experimental setup or intrusive gas sampling. The usefulness of information about the simultaneous spatial distribution of different chemical is illustrated by detecting and identifying the various gases present in a cloud or a plume, and determining the quantity for each of them from the hyperspectral data. Such results illustrate the standoff capability of the Telops' Hyper-Cam for air pollution monitoring.

\section{References}

[1] Farley, V., Chamberland, M., Lagueux, P., Vallières, A., Villemaire, A., Giroux, J., Chemical agent detection and identification with a hyperspectral imaging infrared sensor. Proc. of SPIE, Vol. 6661, pp. 66610L-1-9, 2007.

[2] Tremblay, P. et al., Standoff gas identification and quantification from turbulent stack plumes with an imaging Fourier-transform spectrometer. Proc. of SPIE, Vol. 7673, pp. 76730H-1-12, 2010. 
[3] Sharpe S. W., Johnson T. J., Sams R. L., Chu P. M., Rhoderick G. C., Johnson P. A., Gas-Phase Databases for Quantitative Infrared Spectroscopy. Applied Spectroscopy, 58(12), pp. 1452-1461, 2004.

[4] Savary, S. et al., Standoff identification and quantification of flare emissions using infrared hyperspectral imaging. Proc. of SPIE, Vol. 8024, pp. 80240T$1-8,2010$. 